PROCEEDINGS OF THE

AMERICAN MATHEMATICAL SOCIETY

Volume 140, Number 4, April 2012, Pages 1267-1279

S 0002-9939(2011)10969-9

Article electronically published on July 19, 2011

\title{
INCREASING DIGIT SUBSYSTEMS OF INFINITE ITERATED FUNCTION SYSTEMS
}

\author{
THOMAS JORDAN AND MICHAE RAMS
}

(Communicated by Bryna Kra)

\begin{abstract}
We consider an infinite iterated function system $\left\{f_{i}\right\}_{i=1}^{\infty}$ on $[0,1]$ with a polynomially increasing contraction rate. We look at subsets of such systems where we only allow iterates $f_{i_{1}} \circ f_{i_{2}} \circ f_{i_{3}} \circ \cdots$ if $i_{n}>\Phi\left(i_{n-1}\right)$ for certain increasing functions $\Phi: \mathbb{N} \rightarrow \mathbb{N}$. We compute both the Hausdorff and packing dimensions of such sets. Our results generalise work of Ramharter which shows that the set of continued fractions with strictly increasing digits has Hausdorff dimension $\frac{1}{2}$.
\end{abstract}

\section{INTRODUCTION}

In this paper we consider certain subsets of the attractors of infinite iterated function systems. For each $n \in \mathbb{N}$ we will let $f_{n}:[0,1] \rightarrow[0,1]$ be $C^{1}$ maps such that:

(1) There exists $m \in \mathbb{N}$ and $0<A<1$ such that for all $\left(a_{1}, \ldots, a_{m}\right) \in \mathbb{N}^{m}$ and for all $x \in[0,1]$,

$$
0<\left|\left(f_{a_{1}} \circ \cdots \circ f_{a_{m}}\right)^{\prime}(x)\right| \leq A<1 .
$$

(2) For any $i, j \in \mathbb{N}, f_{i}((0,1)) \cap f_{j}((0,1))=\emptyset$.

(3) There exist $d>1$ such that for any $\varepsilon>0$ there exist $C_{1}(\varepsilon), C_{2}(\varepsilon)>0$ such that for $i \in \mathbb{N}$ there exist constants $\lambda_{i}, \xi_{i}$ such that for all $x \in[0,1]$, $\xi_{i} \leq\left|f_{i}^{\prime}(x)\right| \leq \lambda_{i}$ and

$$
\frac{C_{1}}{i^{d+\varepsilon}} \leq \xi_{i} \leq \lambda_{i} \leq \frac{C_{2}}{i^{d-\varepsilon}} .
$$

We will call such a system a $d$-decaying system.

There will be a natural projection $\Pi: \mathbb{N}^{\mathbb{N}} \rightarrow[0,1]$ defined by

$$
\Pi(\underline{a})=\lim _{n \rightarrow \infty} f_{a_{1}} \circ \cdots \circ f_{a_{n}}(1) .
$$

We will denote $\Lambda=\Pi\left(\mathbb{N}^{\mathbb{N}}\right)$ as the attractor of the system. We will let $T: \Lambda \rightarrow \Lambda$ be the expanding map defined by $T(x)=f_{i}^{-1}(x)$ if $x \in f_{i}([0,1])$. If $x=\Pi(\underline{a})$, then we will refer to $\left\{a_{n}\right\}_{n \in \mathbb{N}}$ as the digits of $x$ (these are not necessarily unique). For brevity of notation for $x \in \Lambda,\left\{a_{i}(x)\right\}_{i \in \mathbb{N}}$ will denote a sequence $\underline{a} \in \mathbb{N}^{\mathbb{N}}$ such that $\Pi(\underline{a})=x$.

Received by the editors October 25, 2010 and, in revised form, December 21, 2010.

2010 Mathematics Subject Classification. Primary 28A80; Secondary 11K50.

The second author's research was supported by grants EU FP6 ToK SPADE2, EU FP6 RTN CODY and MNiSW grant 'Chaos, fraktale i dynamika konforemna'.

(C)2011 American Mathematical Society Reverts to public domain 28 years from publication 
We are interested in the set of $x$ where the digits are increasing monotonically. For a function $\Phi: \mathbb{N} \rightarrow \mathbb{R}$ which satisfies that $\Phi(n) \geq n$ we will denote

$$
X_{\Phi}=\Pi\left\{\underline{a}: a_{n+1}>\Phi\left(a_{n}\right) \text { for all } n \in \mathbb{N}\right\} .
$$

We will be looking at the dimension of these sets for various different notions of dimension. We will be considering Hausdorff dimension, denoted $\operatorname{dim}_{H}$, packing dimension, denoted $\operatorname{dim}_{P}$ and upper box counting dimension denoted $\overline{\operatorname{dim}}_{B}$. For the definitions of these notions of dimension the reader is referred to [F1]. Our motivation for this work is in part to look at iterated function systems with a transient Markov condition; for instance, such systems clearly cannot support an invariant measure. These sets would also have significance when the infinite system is a first return map for a dynamical system $F$ and an interval $J$ since they would represent points where the orbit would take longer and longer to return to the interval $J$. For example if $F$ is the Farey map, $T$ is the Gauss map and $r_{n}(x)$ denotes the $n$th return time of the $F$ orbit of $x$ to $(1 / 2,1)$, then

$$
X_{\Phi}=\left\{x: r_{n+1}(x)>\Phi\left(r_{n}(x)\right\} .\right.
$$

Another aim is to generalise results for the Gauss map to more general systems. Our first result is the following.

Theorem 1.1. Let $\Phi: \mathbb{N} \rightarrow \mathbb{R}$ satisfy that for some $\beta \geq 1$ we have $n \leq \Phi(n) \leq \beta n$ for all $n \in N$. We then have that

$$
\operatorname{dim}_{H} X_{\Phi}=\frac{1}{d} .
$$

Considering packing dimension instead of Hausdorff dimension we obtain the following, stronger result:

Theorem 1.2. Let $s_{0}=\overline{\operatorname{dim}}_{B}\left(\left\{f_{i}(0)\right\}_{i=1}^{\infty}\right)$ and $\Phi: \mathbb{N} \rightarrow \mathbb{R}$ satisfy that $\Phi(n) \geq n$. Then we have that

$$
\operatorname{dim}_{P} X_{\Phi}=\max \left\{s_{0}, \frac{1}{d}\right\} .
$$

To look at $\operatorname{dim}_{H} X_{\Phi}$ for functions $\Phi: \mathbb{N} \rightarrow \mathbb{R}$ where the growth rate is quicker than a linear rate we restrict ourselves to a certain class of $d$-decaying systems. We will call an iterated function system, $\left\{f_{n}\right\}_{n \in \mathbb{N}}$, Gauss-like if

$$
\overline{\bigcup_{i=0}^{\infty} f_{i}([0,1])}=[0,1]
$$

and if for all $x \in[0,1]$ we have that $f_{i}(x)<f_{j}(x)$ implies $i>j$. We then have that

Theorem 1.3. If $\left\{f_{i}\right\}_{i=1}^{\infty}$ is a Gauss-like system, $\alpha>1$ and $\Phi(n)=n^{\alpha}$, then

$$
\operatorname{dim}_{H} X_{\Phi}=\frac{1}{1+\alpha(d-1)} .
$$

Previous work on this type of problem has been done in the case of continued fractions. Here the maps $f_{n}:[0,1] \rightarrow[0,1]$ can be defined by $f_{n}(x)=\frac{1}{x+n}$ for each $n \in \mathbb{N}$. In 1941 Good $\left([\mathbf{G}]\right.$ ) showed that the set where $\lim _{i \rightarrow \infty} a_{i}=\infty$ has dimension $\frac{1}{2}$ and this was extended by Ramharter $[\underline{R}$ to show that the set of $x$ with strictly increasing continued fraction exponents has dimension $\frac{1}{2}$. We will show that this dimension is unchanged if we use the stronger condition $a_{i+1}>\beta a_{i}$ for $\beta>1$ and for all $i \in \mathbb{N}$. However, on the other hand, we will show that if we 
have the condition $a_{i+1}(x)>\left(a_{i}(x)\right)^{\alpha}$ for all $i \in \mathbb{N}$ and $\alpha>1$, then the dimension does drop below $\frac{1}{2}$. Subsequent to the work of Good, several papers, e.g. [L] and WW], have added conditions on the rate of convergence of the $a_{i}$ to infinity either along sequences or subsequences. In particular, Wang and Wu WW] calculate the Hausdorff dimension of the set where $a_{i}(x) \geq \Phi(x)$ for infinitely many $n$ for any function $\Phi$. In Lemma 3.2 in $[\mathrm{FL}$, the Hausdorff dimension of sets, where the value of $a_{n+1}(x)$ is bounded by $s_{n}$ and $N_{S_{n}}$ for a constant $N$ and some sequence $s_{n}$ where $s_{n} \rightarrow \infty$ as $n \rightarrow \infty$ are calculated. In $\mathrm{Mu}$. the dimensions of related sets are considered in the case where each map $f_{i}$ is a similarity.

In this setting of continued fractions, Theorem 1.1 and Theorem 1.3 have the following corollary:

Corollary 1.4. If we denote the continued fraction expansion of $x$ by $a_{1}(x), a_{2}(x)$, $a_{3}(x), \ldots$, then we have that

(1) for any $\beta \geq 1$ we have that

$$
\operatorname{dim}_{H}\left\{x: a_{i+1}(x)>\beta a_{i}(x) \text { for all } i \in \mathbb{N}\right\}=\frac{1}{2} ;
$$

(2) for any $\alpha>1$ we have that

$$
\operatorname{dim}_{H}\left\{x: a_{i+1}(x)>\left(a_{i}(x)\right)^{\alpha} \text { for all } i \in \mathbb{N}\right\}=\frac{1}{1+\alpha} .
$$

It should be noted that in part (1) of the corollary, the case where $\beta=1$ was shown by Ramharter in $[\mathbb{R}$. The second part of this corollary relates to the work by Luczak in $[\mathrm{L}$. Here for $\alpha, \beta>1$ the sets

$$
\Theta[\alpha, \beta]=\left\{x: a_{n}(x) \geq \beta^{\alpha^{n}} \text { for all } n \in \mathbb{N}\right\}
$$

are considered (where $a_{i}(x)$ denote the continued fraction digits of $x$ ). It is shown that $\operatorname{dim} \Theta[\alpha, \beta]=\frac{1}{1+\alpha}$, which corresponds with the dimension found in part (2) of Corollary 1.4. This connection is no surprise since if we have that $a_{i+1}>a_{i}^{\alpha}$ for all $i \in \mathbb{N}$, then $a_{n}(x)>a_{1}(x)^{\alpha^{n}}$.

Finally we can show that Theorem 1.3 does not hold if we consider more general systems. In particular if there are gaps between the first level cylinders, then the situation can be significantly different as illustrated by the following theorem:

Theorem 1.5. For any $d>1$ and any strictly increasing function $\Phi: \mathbb{N} \rightarrow \mathbb{N}$ there exists a d-decaying system $\left\{f_{i}\right\}_{i=1}^{\infty}$ such that

$$
\operatorname{dim}_{H} X_{\Phi}=\frac{1}{d}
$$

Throughout the paper, if $x \in \Lambda$, we will denote the $n$ th-level cylinder containing $x$ by

$$
\mathcal{C}_{n}(x):=f_{a_{1}(x)} \circ \cdots \circ f_{a_{n}(x)}([0,1]) .
$$

The rest of the paper is laid out as follows. In section 2 we prove some lemmas which are the key to the proofs of our main theorems. Theorem 1.1 and Theorem 1.2 are then proved in section 3. Finally, sections 4 and 5 are devoted to the proofs of Theorems 1.3 and 1.5 respectively. 


\section{KEY LEMMAS}

In this section we prove a series of lemmas which will be used in the following sections. We start with two lemmas needed to prove the upper bound for Theorems 1.1 and 1.2. We fix $d>1$ and a $d$-decaying iterated function system $\left\{f_{i}\right\}_{i=1}^{\infty}$. For a positive integer $k$ we will use $\Lambda_{k}$ to denote the attractor of the system $\left\{f_{i}\right\}_{i=k}^{\infty}$.

Lemma 2.1.

$$
\lim _{k \rightarrow \infty} \operatorname{dim}_{H} \Lambda_{k}=\frac{1}{d}
$$

Proof. In the case of the Gauss map this result is contained in the work of Good [G], and the precise asymptotic for the rate of convergence is given in $\mathrm{JK}$. For more general systems it will follow from Bowen's formula for the Hausdorff dimension of infinite iterated function systems given in $\mathrm{MU}$. However some of the systems we are considering do not satisfy the assumptions in [MU], and so we include a proof. First of all we prove that $\operatorname{dim}_{H} \Lambda_{k} \geq \frac{1}{d}$. We fix any $s<\frac{1}{d}$. We can then find $m \in \mathbb{N}$ such that $\sum_{i=k+1}^{m} \xi_{i}^{s} \geq 1$. Consider the iterated function system consisting of the maps $f_{k}, \ldots, f_{m}$ and let $\Lambda_{k}, m \subset \Lambda$, be the attractor. By standard results for iterated function systems, $\operatorname{dim}_{H} \Lambda_{m, k} \geq s_{m}$, where $s_{m}$ is the solution to $\sum_{i=k+1}^{m} \xi_{i}^{s_{m}}=1$, and we know by definition that $s_{m} \geq s$. Since this holds for any $s<\frac{1}{d}$ we know that $\operatorname{dim}_{H} \Lambda \geq \frac{1}{d}$.

To obtain the upper bound we fix $s>\frac{1}{d}$ and choose $k$ such that $\sum_{i=k}^{\infty} \lambda_{i}^{s} \leq 1$. For convenience we will denote $\mathbb{N}(k)$ to be the set of natural numbers greater than or equal to $k$. We get that

$$
\begin{aligned}
\sum_{\left(a_{1}, \ldots, a_{n}\right) \in \mathbb{N}(k)^{n}}\left|a_{1}, \ldots, a_{n}\right|^{s} & \leq \sum_{\left(a_{1}, \ldots, a_{n}\right) \in \mathbb{N}(k)^{n}}\left(\lambda_{a_{1}} \cdots \lambda_{a_{n}}\right)^{s} \\
& \leq\left(\sum_{i=k}^{\infty} \lambda_{i}^{s}\right)^{n} \leq 1 .
\end{aligned}
$$

It then follows that $\operatorname{dim}_{H} \Lambda_{k} \leq s$. Thus for every $s>\frac{1}{d}$ we can find $k$ such that $\operatorname{dim}_{H} \Lambda_{k} \leq s$ since $\operatorname{dim}_{H} \Lambda_{k}$ is clearly monotonically decreasing. The result follows.

We also need an analogue of Lemma 2.1 in terms of upper box dimension or equivalently packing dimension. We will let

$$
s_{0}=\overline{\operatorname{dim}}_{B}\left(\left\{f_{i}(0)\right\}_{i=1}^{\infty}\right) .
$$

Note that instead of 0 we could take any other point of the interval $[0,1]$ and the value of $s_{0}$ would not change.

\section{Lemma 2.2 .}

$$
\lim _{k \rightarrow \infty} \operatorname{dim}_{P} \Lambda_{k} \leq \max \left\{\frac{1}{d}, s_{0}\right\} .
$$

Proof. Let $\varepsilon>0, \delta \ll \varepsilon$ and let $K$ be sufficiently large such that

$$
\sum_{i=K}^{\infty}\left(C_{2}(\delta)\right)^{d^{-1}+\varepsilon} i^{-(d-\delta)\left(d^{-1}+\varepsilon\right)} \leq 1 .
$$

We will fix $K^{-d}<\lambda<1$. We can find a constant $N_{0}>0$ such that for any integer $n \geq 0$ we can cover $\left\{f_{j}(v)\right\}_{j=1}^{\infty}$ by $N_{0} \lambda^{-n\left(s_{0}+\varepsilon\right)}$ intervals of size $\frac{C_{1}(\delta)}{C_{2}(\delta)} \lambda^{(1+2 \delta / d) n}$. 
We let $\mathbb{N}(K)^{*}$ denote all finite words formed from the alphabet $\mathbb{N}(K)$. Let

$$
A_{n}=\left\{\omega \in \mathbb{N}(K)^{*}: \lambda^{n}>\left|f_{\omega}([0,1])\right| \geq \lambda^{n+1}\right\} .
$$

We have that $\# A_{n} \leq \lambda^{-(n+1)\left(d^{-1}+\varepsilon\right)}$. We now fix an integer $N>0$ and find a cover of $\Lambda_{K}$ with intervals of length $\lambda^{N}$. Let $0<n \leq N$ and let $\omega \in A_{n}$. We denote

$$
D(\omega)=\bigcup\left\{f_{\omega} \circ f_{j}([0,1]):\left|f_{\omega} \circ f_{j}([0,1])\right| \leq \lambda^{N}\right\},
$$

where $j \in \mathbb{N}$. We have that

$$
\Lambda_{k} \subset \bigcup_{n=0}^{N} \bigcup_{\omega \in A_{n}} D(\omega) .
$$

We know that $\left\{f_{j}(0)\right\}_{j=1}^{\infty}$ can be covered by at most $N_{0} \lambda^{-(N-n)\left(s_{0}+\varepsilon\right)}$ intervals of size $\frac{C_{1}(\delta)}{C_{2}(\delta)} \lambda^{(1+2 \delta / d)(N-n)}$, and so for $\omega \in A_{n}, D(\omega)$ can be covered by $N_{1} \lambda^{(n-N)\left(s_{0}+\varepsilon\right)}$ intervals of size $\lambda^{-N}$ for a constant $0<N_{1} \leq 3 N_{0}$. Therefore we have that $\Lambda_{K}$ can be covered by

$$
N_{1} \sum_{n=0}^{N} \lambda^{-(n+1)\left(d^{-1}+\varepsilon\right)-(N-n)\left(s_{0}+\varepsilon\right)}
$$

intervals of size $\lambda^{N}$. Thus

$$
\overline{\operatorname{dim}}_{B} \Lambda_{K} \leq \limsup _{n \rightarrow \infty} \frac{\log \sum_{n=0}^{N} \lambda^{-n\left(d^{-1}+\varepsilon\right)-(N-n)\left(s_{0}+\varepsilon\right)}}{-N \log \lambda} \leq \max \left\{d^{-1}, s_{0}\right\}+\varepsilon .
$$

Applying Theorem 3.1 in $\mathrm{MU}$ completes the proof.

We now let $\Phi: \mathbb{N} \rightarrow \mathbb{R}$ satisfy $\Phi(n) \geq n$ for all $n \in \mathbb{N}$ and let the set $X_{\Phi}$ be as defined in (1.1). To prove the lower bounds in Theorems 1.1 and 1.2 we introduce certain subsets of $X_{\Phi}$ which we will use in order to define a measure supported on $X_{\Phi}$. For any natural $n$ let $l(n)$ be the minimal natural number such that

$$
\sum_{i=[\Phi(n)]+1}^{l(n)} \xi_{i}^{1 / d-\varepsilon} \geq 1,
$$

where $[\Phi(n)]$ denotes the integer part of $\Phi(n)$. Let $K$ be the smallest integer such that for any $k \geq K$ we have

$$
k^{-d-\varepsilon} \leq \xi_{k} \leq \lambda_{k} \leq k^{-d+\varepsilon} .
$$

We then define $\left\{l_{n}\right\}_{n \in \mathbb{N}}$ recursively by $l_{1}=K$ and $l_{n+1}=l\left(l_{n}\right)$. Let $Y_{\Phi, \varepsilon}$ be a subset of $X_{\Phi}$ defined as

$$
Y_{\Phi, \varepsilon}=\left\{x \in[0,1]: \Phi\left(l_{n}\right)<a_{n}(x) \leq l_{n+1}\right\} .
$$

Lemma 2.3. There exist $\gamma>1$ such that

$$
\frac{l_{n+1}}{\Phi\left(l_{n}\right)}<\gamma
$$

for all $n$. 
Proof. By assumption we have that $C_{1} i^{-d-\varepsilon} \leq \xi_{i} \leq C_{2} i^{-d+\varepsilon}$. Thus we have that if $n$ is sufficiently large,

$$
2 \geq \sum_{i=\Phi(n)+1}^{l(n)} \xi_{i}^{1 / d-\varepsilon} \geq C_{2} \int_{\Phi(n)+1}^{l(n)} s^{(-d+\varepsilon)(1 / d-\varepsilon)} \mathrm{d} s .
$$

Evaluating this integral and using the fact that for $\varepsilon$ sufficiently small $2\left(d+\frac{1}{d}-\varepsilon\right) \varepsilon<$ 1 , we obtain that

$$
l(n)^{\left(d+\frac{1}{d}-\varepsilon\right) \varepsilon} \leq 2 \Phi(n)^{\left(d+\frac{1}{d}-\varepsilon\right) \varepsilon},
$$

and the result easily follows.

The following lemma is the key to the lower bound for Theorems 1.1, 1.2 and 1.5

Lemma 2.4. We can define a probability measure $\nu$ supported on $Y_{\Phi, \varepsilon}$ such that

(1) $\nu\left(\mathcal{C}_{n}(x)\right)<\left|\mathcal{C}_{n}(x)\right|^{1 / d-\varepsilon}$ for all $x \in Y_{\Phi, \varepsilon}$.

(2) For $\nu$ almost all $x \in Y_{\Phi, \varepsilon}$,

$$
\limsup _{r \rightarrow 0} \frac{\log (\nu(B(x, r)))}{\log r} \geq \frac{1}{d}-\varepsilon .
$$

Proof. We start by fixing a positive integer $n$ and considering the set of integers $I(n):=\left\{\Phi\left(l_{n}\right)+1, \ldots, l_{n+1}\right\}$. We will then refine this set by removing the integers which refer to the left-most and right-most intervals. To be precise let

$$
I^{\prime}(n)=\left\{i \in I(n): \exists j, k \in I(n) \text { with } f_{j}([0,1]) \leq f_{i}([0,1]) \leq f_{k}([0,1])\right\}
$$

(where $J_{1} \leq J_{2}$ is to be understood as: interval $J_{1}$ is to the left of interval $J_{2}$ ). We denote by $s_{n}$ the value such that

$$
\sum_{i \in I^{\prime}(n)} \xi_{i}^{s_{n}}=1
$$

and note that each $s_{n} \geq \frac{1}{d}-\varepsilon$. For each $n$ we will define a finite measure $\mu_{n}$ supported on the finite $\sigma$-algebra given by the sets $\left\{f_{i}([0,1])\right\}_{i \in I^{\prime}(n)}$ and satisfying that $\mu_{n}\left(\mathcal{C}_{\omega_{i}}\right)=\xi_{i}^{s_{n}}$. We can then let $\nu_{n}=\bigotimes_{k=0}^{n-1} \mu_{k} \circ T^{k}$ and note that the extension $\nu$ of these measures (Kolmogorov) will be supported on a subset of $Y_{\beta, \varepsilon}$.

Note that for any cylinder $\mathcal{C}_{\omega_{1} \ldots \omega_{n}}$ we have that

$$
\nu\left(\mathcal{C}_{\omega_{1} \ldots \omega_{n}}\right)=\xi_{\omega_{1}}^{s_{1}} \cdots \xi_{\omega_{n}}^{s_{n}}
$$

and we can immediately deduce (11).

For (2) let $x \in \operatorname{supp}(\mu)$ and fix an $n$. We can then deduce that $a_{n+1}(x) \in I^{\prime}(n)$. Now consider the set of cylinders

$$
Z_{n}=\left\{\Pi\left(\left[a_{1}(x), \ldots, a_{n}(x), j\right]\right)\right\}_{j \in I(n)}
$$

and let $R_{n}=\min _{j \in I(n)} \xi_{a_{1}(x)} \cdots \xi_{a_{n}(x)} \xi_{j}$. We know that $x \in\left[a_{1}(x), \ldots, a_{n}(x), j\right]$ for some $j \in I^{\prime}(n)$. Therefore $B\left(x, R_{n}\right) \subset \mathcal{C}_{n}(x)$ and $B\left(x, R_{n}\right)$ will intersect at most two members of $Z_{n}$. Therefore we have that

$$
\begin{aligned}
\mu\left(B\left(x, R_{n}\right)\right) & \leq 2 C_{2}^{s_{n+1}} \xi_{a_{1}(x)}^{s_{1}} \cdots \xi_{a_{n}(x)}^{s_{n}}\left(\Phi\left(l_{n}\right)+1\right)^{s_{n+1}(-d+\varepsilon)} \\
& \leq 2 \gamma^{-s_{n+1}(-d-\varepsilon)} l_{n+1}^{2 \varepsilon} C_{2}^{s_{n+1}}\left(\xi_{a_{1}(x)} \cdots \xi_{a_{n}(x)}\right)^{1 / d-\varepsilon}\left(l_{n+1}\right)^{s_{n+1}(-d-\varepsilon)} .
\end{aligned}
$$


Thus if we take logarithms we have that

$$
\log \mu\left(B\left(x, R_{n}\right)\right) \leq(1 / d-\varepsilon) \log R_{n}+2 \varepsilon \log l_{n+1}+o\left(-\log R_{n}\right),
$$

and to complete the proof we notice that $-\log l_{n+1} / \log R_{n}$ is uniformly bounded.

\section{Proofs of Theorems 1.1 And 1.2}

Proof of Theorem 1.1. We fix $d>1$, a $d$-decaying system $\left\{f_{i}\right\}_{i=1}^{\infty}$ and a function $\Phi: \mathbb{N} \rightarrow \mathbb{R}$ satisfying $n \leq \Phi(n) \leq \beta n$ for all $n \in \mathbb{N}$ and some $\beta \geq 1$. To prove the upper bound we note that for any $k \in \mathbb{N}$,

$$
X_{\Phi} \subset \bigcup_{l \leq k} \bigcup_{a_{1}<\ldots<a_{l} \leq k} f_{a_{l}} \circ \ldots \circ f_{a_{1}}\left(\Lambda_{k}\right) .
$$

Since the maps $f_{i}$ are bi-Lipschitz, it then follows by Lemma 2.1 that $\operatorname{dim}_{H} X_{\Phi} \leq \frac{1}{d}$.

To compute the lower bound for any $x \in X_{\Phi}$ and $n \in \mathbb{N}$, we let $r_{n}(x):=\left|\mathcal{C}_{n}(x)\right|$. We can freely assume that $\beta$ is strictly greater than 1 (if $\Psi \geq \Phi$, then $X_{\Psi} \subset X_{\Phi}$ ). We then have the following result.

Lemma 3.1. For any $\delta>0$ there exist $l>0$ and $N>0$ such that for any $x, y \in Y_{\Phi, \varepsilon}$ and $n>N$ we have

$$
r_{n}(x)>\left(r_{n+l}(y)\right)^{1+\delta} .
$$

Proof. By applying Lemma 2.3 we can calculate that for any $l \in \mathbb{N}$,

$$
\begin{aligned}
\frac{r_{n}(x)}{\left(r_{n+l}(y)\right)^{1+\delta}} & =\frac{r_{n}(x)}{\left(r_{n}(y)\right)^{1+\delta}} \cdot\left(\frac{r_{n}(y)}{r_{n+l}(y)}\right)^{1+\delta} \\
& \geq\left(\frac{C_{1}(\delta / 2 d)}{\left(C_{2}(\delta / 2 d) \gamma\right)^{1+\delta}}\right)^{n} \cdot \frac{1}{\left(C_{1}(\delta / 2 d)\right)^{l(1+\delta)}} \beta^{n d l(1+\delta)} .
\end{aligned}
$$

Thus if we choose $l$ large enough such that

$$
\beta^{l d(1+\delta)}>\left(C_{1}(\delta / 2 d)\right)^{l(1+\delta)}\left(\frac{\left(C_{2}(\delta / 2 d) \gamma\right)^{1+\delta}}{C_{1}(\delta / 2 d)}\right),
$$

then the proof is complete.

Hence, for any $x \in Y_{\Phi, \varepsilon}, n>N$, and $\left(r_{n+l+1}(x)\right)^{1+\delta} \leq r \leq\left(r_{n+l}(x)\right)^{1+\delta}$, the set $B_{r}(x) \cap Y_{\Phi, \varepsilon}$ will be contained in $\mathcal{C}_{n}(x) \cup \mathcal{C}_{n}(y)$ for some $y \in Y_{\Phi, \varepsilon}$. We also have $r \geq\left(r_{n+l+1}(x)\right)^{1+\delta}>\left(r_{n+2 l+1}(y)\right)^{(1+\delta)^{2}}$. Thus we will have

$$
\liminf _{r \rightarrow 0} \frac{\log \nu\left(B_{r}(x)\right)}{\log r} \geq \liminf _{n \rightarrow \infty} \inf _{y \in Y_{\beta, \varepsilon}} \frac{(1 / d-\varepsilon) \log r_{n}(y)+\log 2}{(1+\delta)^{2} \log r_{n+2 l+1}(y)} .
$$

The only thing missing in the proof of Theorem 1.1 is a comparison of sizes of $r_{n}(x)$ and $r_{n+1}(x)$.

Lemma 3.2. There exists a sequence $v_{n} \rightarrow 1$ such that for every $x \in Y_{\beta, \varepsilon}$,

$$
\frac{\log r_{n+1}(x)}{\log r_{n}(x)}<v_{n}
$$


Proof. We have that

$$
r_{n+1}(x) \geq \xi_{a_{n+1}(x)} r_{n}(x) .
$$

Thus it suffices to show that $\frac{\log \xi_{a_{n+1}(x)}}{\log r_{n}(x)}$ tends to 0 uniformly in $x$. For $\varepsilon>0$ we have that for all $x$,

$$
r_{n}(x) \leq \prod_{i=1}^{n} \frac{C_{2}}{i^{d-\varepsilon}} \leq \frac{C_{2}^{n}}{(n !)^{d-\varepsilon}} .
$$

On the other hand, by Lemma 2.3 and the definition of $\Phi$,

$$
l_{n+1} \leq \gamma \Phi\left(l_{n}\right) \leq \beta \gamma l_{n} .
$$

Thus $a_{n+1}(x) \leq(\beta \gamma)^{n}$ and so $\xi_{a_{n+1}(x)} \geq(\beta \gamma)^{-n(d+\varepsilon)}$ and the result follows.

Proof of Theorem 1.2. We fix $d>1$, a $d$-decaying system $\left\{f_{i}\right\}_{i=1}^{\infty}$ and a function $\Phi: \mathbb{N} \rightarrow \mathbb{R}$ such that $\Phi(n) \geq n$. We will let $s_{0}=\overline{\operatorname{dim}}_{B}\left(\left\{f_{i}(0)\right\}_{i=1}^{\infty}\right)$. To show that $\operatorname{dim}_{P} X_{\Phi} \leq \min \left\{1 / d, s_{0}\right\}$ we simply replicate the upper bound in the proof of Theorem 1.1 with Lemma 2.2 replacing Lemma 2.1. The fact that $\operatorname{dim}_{P} X_{\Phi} \geq \frac{1}{d}$ can immediately be deduced from Lemma 2.4.

We now turn to the case where $s_{0} \geq \frac{1}{d}$. First we need to show that the upper box counting dimension and the packing dimension of $X_{\Phi}$ are the same.

Lemma 3.3. We have that for any function $\Phi: \mathbb{N} \rightarrow \mathbb{N}$ with $\Phi(n) \geq n$,

$$
\operatorname{dim}_{P} X_{\Phi}=\overline{\operatorname{dim}}_{B} X_{\Phi}
$$

Proof. It can easily be seen that the proof of Theorem 3.1 in $\mathrm{MU}$ can be applied in this situation.

We let $J$ denote the closure of $X_{\Phi}$ and note that by Lemma 3.3 we can deduce that $\operatorname{dim}_{P} X_{\Phi}=\overline{\operatorname{dim}}_{B} J$. We will let $v$ be some accumulation point of $\left\{f_{i}(1)\right\}$. We then have that $J \supset\left\{f_{i}(v)\right\}_{i=1}^{\infty}$ and so $\overline{\operatorname{dim}}_{B} J \geq s_{0}$ and the result immediately follows by Lemma 3.3 .

\section{Proof of Theorem 1.3}

We fix $d>1$, a Gauss-like $d$-decaying system $\left\{f_{i}\right\}_{i=1}^{\infty}, \alpha>1$ and let $\Phi(n)=n^{\alpha}$. Denote $s=1 /(1+\alpha(d-1))$. It is enough to prove that for every $K>1$,

$$
\operatorname{dim}_{H} X_{\Phi, K}=\frac{1}{1+\alpha(d-1)}
$$

where

$$
X_{\Phi, K}=\left\{x \in X_{\Phi}: a_{1}(x)=K\right\}
$$

Indeed, we have

$$
X_{\Phi, K} \subset X_{\Phi}=\left\{x_{0}\right\} \cup \bigcup_{n=0}^{\infty} \bigcup_{K=2}^{\infty} f_{1}^{n} X_{\Phi, K},
$$

where $x_{0}$ is the fixed point of $f_{1}$. We fix $K>1, \delta>0$ and denote $C_{1}=C_{1}(\delta), C_{2}=$ $C_{2}(\delta)$.

Given $x \in X_{\Phi}$ we define

$$
\Delta_{n}(x)=\bigcup\left\{\mathcal{C}_{n+1}(y): y \in \mathcal{C}_{n}(x) \cap X_{\Phi}\right\} .
$$


Obviously, it is the union of all $(n+1)$-st level subcylinders of $\mathcal{C}_{n}(x)$, where the $(n+1)$-st coordinate is at least $a_{n}(x)^{\alpha}$. We have

$$
C_{1}^{n} \prod_{i=1}^{n} a_{i}(x)^{-d-\delta} \leq\left|\mathcal{C}_{n}(x)\right| \leq C_{2}^{n} \prod_{i=1}^{n} a_{i}(x)^{-d+\delta}
$$

and

$$
\begin{aligned}
C_{1}^{n+1} C_{4}^{-1} a_{n}(x)^{-(d+\delta-1) \alpha} \prod_{i=1}^{n} a_{i}(x)^{-d-\delta} & \leq\left|\Delta_{n}(x)\right| \\
& \leq C_{2}^{n+1} C_{4} a_{n}(x)^{-(d-\delta-1) \alpha} \prod_{i=1}^{n} a_{i}(x)^{-d+\delta} .
\end{aligned}
$$

We will distribute on $X_{\Phi, K}$ a probabilistic measure $\mu$, satisfying $\mu\left(a_{1}(x)=K\right)=1$ and

$$
\mu\left(a_{n+1}(x)=j \mid a_{n}(x)=i\right)= \begin{cases}0 & \text { if } j<i^{\alpha}, \\ c_{i} i^{\alpha(d-1) s} j^{-(d+\alpha(d-1)) s} & \text { if } j>i^{\alpha}\end{cases}
$$

where

$$
c_{i}=\frac{1}{\sum_{j>i^{\alpha}} i^{\alpha(d-1) s} j^{-(d+\alpha(d-1)) s}}
$$

is a normalising constant. It is easy to check that for some $C_{3}>1$ we have

$$
C_{3}^{-1} \leq c_{i} \leq C_{3}
$$

for all $i$ (in fact, $c_{i} \rightarrow(d+\alpha(d-1)) s+1$ as $\left.i \rightarrow \infty\right)$.

The reason we have chosen the measure $\mu$ in this way is that for all $x \in X_{\Phi, K}$, we have for each $n$,

$$
\begin{aligned}
& C_{3}^{-n} \prod_{i=2}^{n} a_{i}(x)^{-d s} \cdot a_{1}(x)^{\alpha(d-1) s} a_{n}(x)^{-\alpha(d-1) s} \leq \mu\left(\Delta_{n}(x)\right)=\mu\left(\mathcal{C}_{n}(x)\right) \\
& \leq C_{3}^{n} \prod_{i=2}^{n} a_{i}(x)^{-d s} \cdot a_{1}(x)^{\alpha(d-1) s} a_{n}(x)^{-\alpha(d-1) s} .
\end{aligned}
$$

Comparing this with (4.1) we have that for all $x \in X_{\Phi, K}$,

$$
C_{6}^{-1} C_{5}^{-n}\left|\Delta_{n}(x)\right|^{(1+c \delta) s} \leq \mu\left(\Delta_{n}(x)\right) \leq C_{6} C_{5}^{n}\left|\Delta_{n}(x)\right|^{(1-c \delta) s} .
$$

Note that

$$
\left|\Delta_{n}(x)\right|<\left|\mathcal{C}_{n}(x)\right| \leq \prod_{i=1}^{n} C_{2} K^{-(d-\delta) \alpha^{i-1}}=C_{2}^{n} K^{-(d-\delta)\left(\alpha^{n}-1\right) /(\alpha-1)} .
$$

Hence for $x \in X_{\Phi, K}$ we can calculate

$$
\begin{aligned}
\frac{\log \mu\left(B_{\left|\Delta_{n}(x)\right|}(x)\right)}{\log \left|\Delta_{n}(x)\right|} & \leq \frac{\log \mu\left(\Delta_{N}(x)\right)}{\log \left|\Delta_{n}(x)\right|} \\
& \leq s(1+c \delta)+\frac{o\left(-\log \left|\Delta_{n}(x)\right|\right)}{\log \left|\Delta_{n}(x)\right|}
\end{aligned}
$$

Thus we can conclude that

$$
\operatorname{dim}_{H} X_{\Phi, K} \leq s(1+c \delta)
$$


For the lower bound on the Hausdorff dimension we will use the Frostman Lemma again. Denote

$$
r_{n}(x)=\left|\Delta_{n}(x)\right|, R_{n}(x)=\left|\mathcal{C}_{n}(x)\right| .
$$

We already know that

$$
\lim _{n \rightarrow \infty} \frac{\log \mu\left(\Delta_{n}(x)\right)}{\log \left|\Delta_{n}(x)\right|} \geq s(1-c \delta) .
$$

$B_{r_{n}(x)}(x)$ contains $\Delta_{n}(x)$ and might intersect at most one other $\Delta_{n}(y)$. Moreover, this $\Delta_{n}(y)$ must be a neighbouring one, which means that $a_{i}(x)=a_{i}(y)$ for $i<n$ and $\left|a_{n}(x)-a_{n}(y)\right|=1$. Hence, by (4.3) we have that

$$
\mu\left(B_{r_{n}(x)}(x)\right) \leq(2+\varepsilon) C_{6} C_{5}^{n} r_{n}(x)^{(1-c \delta) s} .
$$

We then have that

$$
\log \mu\left(B_{r_{n}(x)}(x)\right) \leq(1-c \delta) s \log r_{n}+o\left(-\log r_{n}\right) .
$$

We need to use this estimate to find $\frac{\log \mu\left(B_{r}(x)\right)}{\log r}$ for $r_{n}(x)<r<R_{n}(x)$ and $R_{n+1}(x)<r<r_{n}(x)$. The first of these ranges is easy: each $\mathcal{C}_{n}(x) \backslash \Delta_{n}(x)$ has length comparable to $\left|\mathcal{C}_{n}(x)\right|$. Hence, the ball $B_{r}(x)$ for $r_{n}(x)<r<R_{n}(x)$ will be much bigger than $B_{r_{n}(x)}(x)$ but will still intersect at most $\Delta_{n}(x)$ plus one more $\Delta_{n}(y)$. So, in this range

$$
\frac{\log \mu\left(B_{r}(x)\right)}{\log r} \geq(1-c \delta) s-o(1) .
$$

In the range $R_{n+1}(x)<r<r_{n}(x)$ the ball $B_{r}(x)$ will actually intersect several $\mathcal{C}_{n+1}(y), y \in X_{\Phi, K}$. Let us define

$$
D_{r}(x)=\bigcup\left\{\mathcal{C}_{n+1}(y): y \in X_{\Phi, K} \cap B_{r}(x)\right\} .
$$

Note that $\mu\left(D_{r}(x)\right) \geq \mu\left(B_{r}(x)\right)$ but $\left|D_{r}(x)\right| \leq 2 C_{2}^{n+1} / C_{1}^{n+1} r^{1-\delta}$. Hence, we can use $D_{r}(x)$ instead of $B_{r}(x)$ to estimate the local dimension of $\mu$ at $x$, and the estimation will change at most by a factor $(1 \pm \delta)$.

The set $\mathcal{D}=D_{r}(x)$ is a union of consecutive $(n+1)$-st level cylinders $\mathcal{C}_{n+1}(y)$ with $a_{i}(y)=a_{i}(x)$ for $i \leq n$ and $l_{1} \leq a_{n+1}(y) \leq l_{2}$, where $l_{1} \geq a_{n}(x)^{\alpha}$ and $l_{2} \leq \infty$. We have $\mathcal{C}_{n}(x)=\bigcup_{i=l_{1}}^{l_{2}} \mathcal{C}_{n+1}\left(y_{i}\right)$ (where $y_{i}$ is a point from $\mathcal{C}_{n}(x) \cap X_{\Phi, K}$ with $(n+1)$-st symbol in the symbolic expansion equal to $i)$. We have

$$
\left|\mathcal{C}_{n+1}\left(y_{i}\right)\right| \geq i^{-d}\left|\mathcal{C}_{n}(x)\right|^{1+c \delta}
$$

hence

$$
|\mathcal{D}| \geq\left|\mathcal{C}_{n}(x)\right|^{1+c \delta} \sum_{i=l_{1}}^{l_{2}} i^{-d} \approx\left(l_{1}^{-(d-1)}-l_{2}^{-(d-1)}\right)\left|\mathcal{C}_{n}(x)\right|^{1+c \delta} .
$$

We also have

$$
\left|\Delta_{n+1}\left(y_{i}\right)\right| \leq\left|\mathcal{C}_{n}(x)\right|^{1-c \delta} i^{-d-\alpha(d-1)}
$$

hence by (4.3),

$$
\mu(\mathcal{D})=\sum_{i=l_{1}}^{l_{2}} \mu\left(\Delta_{n+1}\left(y_{i}\right)\right) \leq C_{6} C_{5}^{n+1}\left|\mathcal{C}_{n}(x)\right|^{(1-2 c \delta) s} \sum_{i=l_{1}}^{l_{2}} i^{-(d+\alpha(d-1)) s(1-c \delta)} .
$$


Note that

$$
\begin{aligned}
\sum_{i=l_{1}}^{l_{2}} i^{-(d+\alpha(d-1)) s(1-c \delta)} & \leq l_{2}^{(d+\alpha(d-1)) s c \delta} \cdot \sum_{i=l_{1}}^{l_{2}} i^{-(d+\alpha(d-1)) s} \\
& \leq|\mathcal{D}|^{-c \alpha \delta} \cdot \sum_{i=l_{1}}^{l_{2}} i^{-(d+\alpha(d-1)) s} .
\end{aligned}
$$

Thus we have that

$$
\begin{aligned}
\log \mu(\mathcal{D}) \leq & \log \left(\left|\mathcal{C}_{n}(x)\right|^{(1-2 c \delta) s} \sum_{i=l_{1}}^{l_{2}} i^{-(d+\alpha(d-1)) s}\right)-c \alpha \delta \log |\mathcal{D}|+o(-\log (|\mathcal{D}|)) \\
\approx & \log \left(\left(l_{1}^{-(d+\alpha(d-1)) s+1}-l_{2}^{-(d+\alpha(d-1)) s+1}\right)\left|\mathcal{C}_{n}(x)\right|^{(1-2 c \delta) s}\right) \\
& -c \alpha \delta \log |\mathcal{D}|+o(-\log (|\mathcal{D}|)) \\
= & \log \left(\left(l_{1}^{-(d-1) s}-l_{2}^{-(d-1) s}\right)\left|\mathcal{C}_{n}(x)\right|^{s}\right)+o(-\log (|\mathcal{D}|))
\end{aligned}
$$

where we use that

$$
(d+\alpha(d-1)) s-1=(d-1) s .
$$

By the concavity of the function $x \rightarrow x^{s}$ for $s<1$, we have that

$$
a=b^{s} \wedge c=d^{s} \Longrightarrow(a-c) \leq(b-d)^{s} .
$$

Hence we can conclude that

$$
\log (\mu(\mathcal{D})) \leq s(1-(3+\alpha) c \delta) \log |\mathcal{D}|+o(-\log |\mathcal{D}|),
$$

and the proof is complete.

\section{Proof of Theorem 1.5}

We start by fixing an increasing function $\Phi: \mathbb{N} \rightarrow \mathbb{N}$ and $d>1$. We need to find a $d$-decaying system $\left\{f_{i}\right\}_{i=1}^{\infty}$ such that

$$
\operatorname{dim}_{H} X_{\Phi}=\frac{1}{d}
$$

We will fix $\varepsilon>0$. As in section 3, we define by $l(n)$ the smallest number for which

$$
\sum_{i=\Phi(n)+1}^{l(n)} C^{1 / d-\varepsilon} i^{-1+d \varepsilon} \geq 1 .
$$

We define $l_{1}=1$ and $l_{n+1}=l\left(l_{n}\right)$. As in Lemma 2.3. we have that

$$
l_{n+1}<\gamma \Phi\left(l_{n}\right)
$$

for some $\gamma>1$.

The system will be piecewise linear of the form $T_{i}(x)=\frac{C}{i^{d}} x+a_{i}$. We will have that

$$
C=\frac{1}{\sum_{i=1}^{\infty} i^{-d}+\sum_{n=1}^{\infty} n^{-2} l_{n+1}^{-1}\left(l_{n+1}-\Phi\left(l_{n}\right)\right)} .
$$

We define the constants $a_{i}$ recursively by letting $a_{1}=1-c i^{-d}$ and let

$$
a_{n}=\left\{\begin{array}{lll}
a_{n-1}-C n^{-d} & \text { if } & n \notin\left(\Phi\left(l_{n}\right), l_{n+1}\right) \text { for any } n \in \mathbb{N}, \\
a_{n-1}-C n^{-d}-C j^{-2} l_{j+1}^{-1} & \text { if } & n \in\left(\Phi\left(l_{j}\right), l_{j+1}\right) \text { for some } j \in \mathbb{N} .
\end{array}\right.
$$


As in section 3 and Lemma 2.4 we can define

$$
\tilde{X}_{\Phi}=\left\{x: \Phi\left(l_{n-1}\right)+1 \leq a_{n}(x) \leq l_{n}(x)\right\}
$$

and distribute on $\tilde{X}_{\Phi}$ a measure $\nu$ such that

$$
\nu\left(\mathcal{C}_{n}(x)\right) \leq\left|\mathcal{C}_{n}(x)\right|^{(1 / d-\varepsilon)}
$$

for all $x \in \tilde{X}_{\Phi}$. For $x \in \tilde{X}_{\Phi}$, let $Z_{n}(x)$ denote the minimal interval containing $\mathcal{C}_{n}(x) \cap \tilde{X}_{\Phi}$. We can calculate

$$
\begin{aligned}
\left|Z_{n}(x)\right| & \approx\left|\mathcal{C}_{n}(x)\right|\left(C n^{-2} l_{n+1}^{-1}\left(l_{n+1}-\Phi\left(l_{n}\right)\right)+\sum_{i=\Phi\left(l_{n}\right)}^{l_{n+1}} i^{-d}\right) \\
& \approx n^{-2}\left|\mathcal{C}_{n}(x)\right| .
\end{aligned}
$$

We can calculate that for any cylinder $\mathcal{C}_{n}(x), i \neq j \in\left(\Phi\left(l_{n}\right), l_{n}+1\right]$ the cylinders $\mathcal{C}_{n+1}\left(y_{i}\right)$ and $\mathcal{C}_{n+1}\left(y_{j}\right)$ will be separated by a gap of length at least $C n^{-2} l_{n+1}^{-1}$.

Hence, for $r_{n+1}<r \leq r_{n}$,

$$
\mu\left(B_{r}(x)\right) \leq g_{n}(r)=\left(1+c r \frac{n^{2}\left(l_{n+1}-\Phi\left(l_{n}\right)\right)}{r_{n}}\right) r_{n+1}^{1 / d-\varepsilon}
$$

(where $r_{n}=\left|\mathcal{C}_{n}(x)\right|$ ). Note that

$$
g_{n}(r) \leq c r^{1 / d-\varepsilon}
$$

for $r=r_{n}$ and for $r=r_{n+1}$, and $g_{n}(r)$ is a linear function in between. As $x \rightarrow x^{1 / d}$ is a concave function, we have

$$
g_{n}(r)<c r^{1 / d-\varepsilon}
$$

for $r_{n+1}<r<r_{n}$. Hence,

$$
\liminf _{r \rightarrow 0} \frac{\log \mu\left(B_{r}(x)\right)}{\log r} \geq \frac{1}{d}-\varepsilon
$$

and the proof is complete.

\section{ACKNOWLEDGEMENTS}

We would like to thank Omri Sarig and Marc Kesseböhmer for subsequent useful discussions. In particular we would like to thank Marc Kesseböhmer for informing us of the work of Ramharter, of which we were previously unaware.

\section{REFERENCES}

[F1] K.J. Falconer, Fractal geometry. Mathematical foundations and applications, John Wiley, 1990. MR $1102677(92 \mathrm{j}: 28008)$

[FL] Al-Hua Fan, Ling-Min Liao, Bao-Wei Wang and Jun Wu, On Khintchine exponents and Lyapunov exponents of continued fractions, Ergod. Th. ES Dynam. Sys. 29 (2009), 73-109. MR 2470627 (2009m:11124)

[G] I. J. Good, The fractional dimensional theory of continued fractions, Proc. Cambridge Philos. Soc. 37 (1941), 199-228. MR0004878(3:75b)

[JK] Johannes Jaerisch and Marc Kesseböhmer, The arithmetic-geometric scaling spectrum for continued fractions, Arkiv för Matematik 48 (2010), no. 2, 335-360. MR2672614

[L] Tomasz Łuczak, On the fractional dimension of sets of continued fractions, Mathematika 44 (1997), no. 1, 50-53. MR.1464375 (98i:11059a)

[MU] D. Mauldin and M. Urbanski, Dimensions and measures in infinite iterated function systems, Proc. London Math. Soc. (3) 73 (1996), no. 1, 105-154. MR1387085 (97c:28020) 
[McM] Curt McMullen, Area and Hausdorff dimension of Julia sets of entire functions, Trans. Amer. Math. Soc. 300 (1987), 329-342. MR871679 (88a:30057)

[Mu S. Munday, A note on Diophantine-type fractals for $\alpha$-Lüroth systems, to appear in Integers: Electronic Journal of Combinatorial Number Theory.

[R] G. Ramharter, Eine Bemerkung über gewisse Nullmengen von Kettenbrüchen. Ann. Univ. Sci. Budapest. Eötvös Sect. Math. 28 (1985), 11-15. MR856971(88a:11072)

[WW] Bao-Wei Wang and Jun Wu, Hausdorff dimension of certain sets arising in continued fraction expansions, Adv. Math. 218 (2008), no. 5, 1319-1339. MR2419924 (2009d:11115)

School of Mathematics, The University of Bristol, University Walk, Clifton, BrisTOL, BS8 1TW, United Kingdom

E-mail address: thomas.jordan@bristol.ac.uk

Institute of Mathematics, Polish Academy of Sciences, ul. Śniadeckich 8, 00-956 WARSZAWA, Poland

E-mail address: M.Rams@impan.gov.pl 